\title{
Comparison of Clinical Pregnancy Rates and Implantation Rates in Hysteroscopic Lateral Metroplasty versus Endometrial Scratching in Patients of Repeated Implantation Failures
}

\author{
Ashish Kale ${ }^{1}$, Ashwini Kale ${ }^{2}$
}

\begin{abstract}
Introduction: Implantation is one of the rate-limiting steps of success rates in patients undergoing in vitro fertilization. Repeated implantation failure is defined as failure of good-quality embryos to successfully implant in uterine cavity in repeated IVF treatment cycle. The cause of such implantation failures may be maternal or embryonal. Endometrial scratching and lateral metroplasty are found to increase successful implantation rates in various studies. There is hardly any study in the literature comparing the success rates of endometrial scratching and hysteroscopic lateral metroplasty. Keeping in mind this fact, we conducted this comparative study to compare the successful pregnancy rates and clinical pregnancy rates in patients who had undergone endometrial scratching and hysteroscopic lateral metroplasty for repeated implantation failures. Materials and methods: A total of 60 patients having primary or secondary infertility and had history of repeated implantation failure were included in this study on the basis of a predefined inclusion and exclusion criteria. The patients were divided into two groups on the basis of treatment they received. In group A, 30 patients underwent endometrial scratching, and in group B, 30 patients underwent hysteroscopic lateral metroplasty. The successful pregnancy rates and clinical pregnancy rates in both the groups were compared. SPSS 22.0 was used for statistical analysis, and $P$ value less than 0.05 was taken as statistically significant.

Results: Out of 60 patients, $50(87.5 \%)$ patients belonged to primary infertility, whereas $10(12.5 \%)$ patients were found to have secondary infertility. The mean age of patients in group A was $34.12 \pm 3.12$, whereas the mean age in group B was $35.42 \pm 4.46$ years. The mean duration of infertility in patients of group $A$ and group $B$ was found to be $7.43 \pm 2.50$ and $6.93 \pm 2.54$ years, respectively. The mean number of antral follicle count in group A was $9.52 \pm 1.82$, whereas in group B, it was $10.42 \pm 1.52$. In group A, $12(40 \%)$ patients were confirmed to be having successful pregnancy on the basis of $\beta$-hCG levels on D14, whereas $17(56.67 \%)$ patients in group B were found to have successful pregnancy on the basis of $\beta$-hCG. Fetal cardiac activity was present in $13(43.33 \%)$ patients and $18(60 \%)$ patients in group A and group B, respectively $(p=0.301)$. The difference was not found to be statistically significant $(p=0.30)$.

Conclusion: Endometrial scratching and hysteroscopic lateral metroplasty both are effective management strategies in patients having history of repeated implantation failure. Though lateral metroplasty appears to be a better choice, the difference was statistically insignificant $(p>0.05)$. Keywords: Clinical pregnancy rates, Endometrial scratching, Lateral metroplasty, Repeated implantation failure. Journal of South Asian Federation of Obstetrics and Gynaecology (2020): 10.5005/jp-journals-10006-1835
\end{abstract}

\section{INTRODUCTION}

Successful implantation is one of the most crucial steps of not only in vitro fertilization but also patients undergoing artificial insemination. One of the important causes of repeated failure of in vitro fertilization experienced by infertile couples is failed implantation. ${ }^{1}$ Many IVF specialists have been frustrated by the fact that in many patients undergoing assisted reproductive techniques such as intracytoplasmic sperm injection and intrauterine insemination when everything appears to be going smoothly, there is implantation failure. In a sense, failure of implantation appears to be one of the most important rate-limiting steps in in vitro fertilization. Repeated implantation failure is one of the major causes due to which even in best IVF centers the success rates of IVF have been reduced to less than $30 \%{ }^{2}$

Repeated implantation failure is defined as failure of goodquality embryos to successfully implant in uterine cavity in repeated IVF treatment cycle. Causes responsible for implantation failure may be divided into maternal and embryonic factors. ${ }^{3}$ The common maternal factors responsible for implantation failure may include congenital uterine anomalies (bicornuate, septate, or hypoplastic uterus), immunological factors (presence of autoantibodies and couples sharing common HLA alleles), thrombophilia, and

\begin{abstract}
1,2Department of Obstetrics and Gynecology, Ashakiran Hospital and Asha IVF Center, Pune, Maharashtra, India

Corresponding Author: Ashish Kale, Department of Obstetrics and Gynecology, Ashakiran Hospital and Asha IVFCenter, Pune, Maharashtra, India, Phone: +91 20 24458626, e-mail: drashishkale1978@yahoo.com How to cite this article: Kale A, Kale A. Comparison of Clinical Pregnancy Rates and Implantation Rates in Hysteroscopic Lateral Metroplasty versus Endometrial Scratching in Patients of Repeated Implantation Failures. J South Asian Feder Obst Gynae 2020;12(6):348-352.
\end{abstract}

Source of support: Nil

Conflict of interest: None

unfavorable endometrium (thin endometrium, nonreceptive endometrium). ${ }^{4}$ Implantation failure may also be due to embryonal factors such as chromosomal anomalies, mosaicism, chromosomal inversions, and deletion that may be responsible for implantation failure. ${ }^{5}$

Endometrial scratching as a treatment for repeated implantation failure was first attempted by Barash et al. who found that after multiple endometrial scratching, there was a statistically significant increase in implantation rates. Since then, endometrial scratching 
has been frequently used in various in vitro fertilization centers across the globe. Various investigators have used various devices for endometrial scratching that includes endometrial biopsy catheter, Karman cannula, and Novak curette. Though the exact mechanism by which repeated endometrial injury or scratching increases successful implantation rate is not clear, there appears to be a convincing evidence of its benefits in the treatment of repeated implantation failure. This procedure has been frequently used and usually is well tolerable. Mild pain may be experienced by the patients, which may sometimes need analgesics. The complications such as endometritis and perforation may be seen, but they are very rare in experienced hands. ${ }^{6}$

Another procedure that has been reported to be associated with increased implantation rates by various researchers is hysteroscopic lateral metroplasty. This procedure consists of electrosurgical incision of the lateral walls of the uterine cavity using a Collins knife. There are various studies that have reported increase in success rates of in vitro fertilization in women who had repeated cycle failures due to implantation failure. The complications such as infection, hemorrhage, and perforation are rare but may occur. ${ }^{7}$

Though there are various studies that have analyzed the success rates after undertaking interventions such as endometrial scratching and hysteroscopic metroplasty, there is hardly any study comparing the success rates of uterine scratching and hysteroscopic metroplasty. Keeping in mind the scarcity of comparative studies of these two interventions, we conducted this comparative study of patients undergoing uterine scratching and hysteroscopic metroplasty for repeated implantation failure.

\section{Materials and Methods}

This was a comparative study conducted in a tertiary care IVF center situated in an urban area. In this study, 60 women with either primary or secondary infertility who had history of repeated implantation failure ( 3 or more) were included on the basis of a predefined inclusion and exclusion criteria. These 60 patients were divided into two groups on the basis of treatment they would receive. Demographic details such as age of the patients, time since marriage, menstrual history, type of infertility (primary or secondary infertility), and reports of hormonal assessment were noted down in a proforma. Reports of ultrasound, Doppler examination, hysterosalpingography, as well as any other imaging technique were also noted down. Presences of any anatomical abnormalities of ovaries, uterus, or fallopian tubes were specifically looked for in previous imaging if available. Any significant past history (such as autoimmune diseases, Koch's and hematological disorders) and history of any systemic illness (diabetes, hypertension, or asthma) were also asked for and noted down. A baseline progesterone, estradiol, luteinizing hormone, and follicle-stimulating hormone levels were done in all the cases. Patients were divided into two groups on the basis of intervention.

Group A patients underwent uterine scratching on day 21 of the previous cycle, whereas group $B$ patients underwent lateral metroplasty on day 7 of the previous cycle. Embryo transfer was done when endometrium thickness was more than $8 \mathrm{~mm}$ and vascularity (grade 3 ) was noted. The successful pregnancy was diagnosed on the basis of $\beta$-hCG levels on day 14 of transfer and confirmed on the basis of fetal cardiac activity later in pregnancy (10 weeks). Successful pregnancy rates in both the groups were compared. The statistical analysis was done using SPSS 22.0 software, and for all statistical purposes, $P$ value less than 0.05 was taken as statistically significant.

\section{InCLUSION Criteria}

- Women having primary or secondary infertility and had history of repeated implantation failure (three or more failed implantations).

- Given informed consent to be part of study.

- Age between 30 and 38 years.

- Thin endometrium $(<7 \mathrm{~mm})$ at the time of trigger.

- Patient who received medical treatment for thin endometrium like ethinyl estradiol $8 \mathrm{mg}$ and those with poor vascularity who received vaginal sildenafil $25 \mathrm{mg}$ TDS not showing significant response in the cycle.

\section{Exclusion Criteria}

- Those who refused consent.

- Those with congenital uterine anomalies.

- Uterine synechia or Asherman's syndrome diagnosed on the basis of previous imaging.

- Thin endometrium due to endometritis.

- Patients who started responding to medical line of management.

- Patients with systemic illnesses, autoimmune disorders and those predisposed for endometritis.

\section{Results}

This was a comparative study in which 60 patients with either primary or secondary infertility and who had history of repeated transplantation failure were included. Out of 60 patients, $50(87.5 \%)$ patients belonged to primary infertility, whereas $10(12.5 \%)$ patients were found to have secondary infertility (Fig. 1).

The analysis of age-groups of the studied cases showed that the mean age of patients in group $A$ was $34.12 \pm 3.12$, whereas the mean age in group $B$ was $35.42 \pm 4.46$ years. The mean age of patients in both the groups was found to be comparable with no statistically significant difference in the age of patients in both the groups $(P>0.05)$ (Table 1).

The analysis of the duration of the infertility in the group A cases showed that 18 patients (50\%) had history of infertility between 6

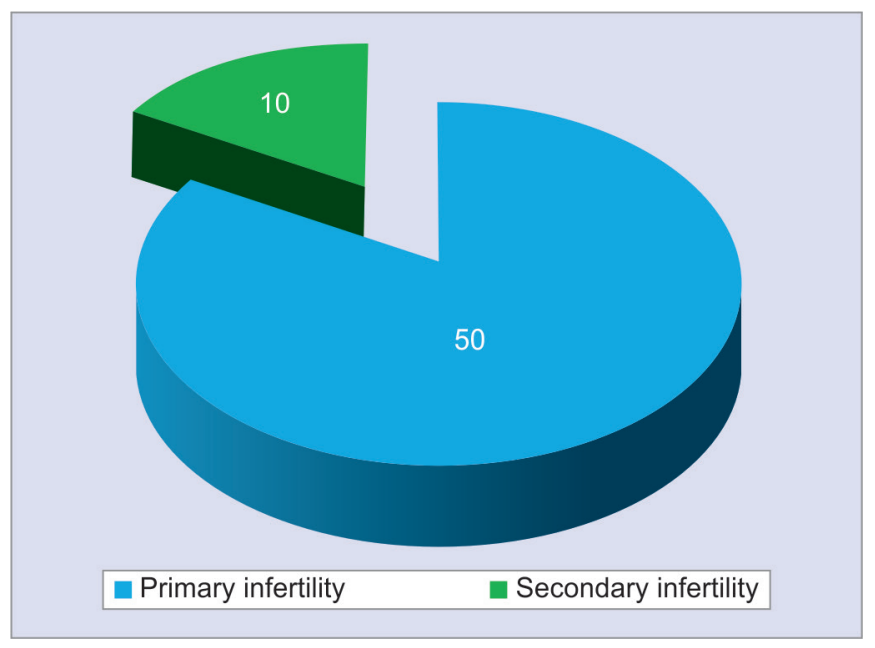

Fig. 1: Primary versus secondary infertility in the studied cases 
Table 1: Comparison of mean age of the studied cases

\begin{tabular}{llll}
\hline Study group & Mean age (years) & Std. deviation & Significance \\
\hline Group A & 34.12 & 3.12 & $P=0.196$ \\
Group B & 35.42 & 4.46 & Not significant \\
\hline
\end{tabular}

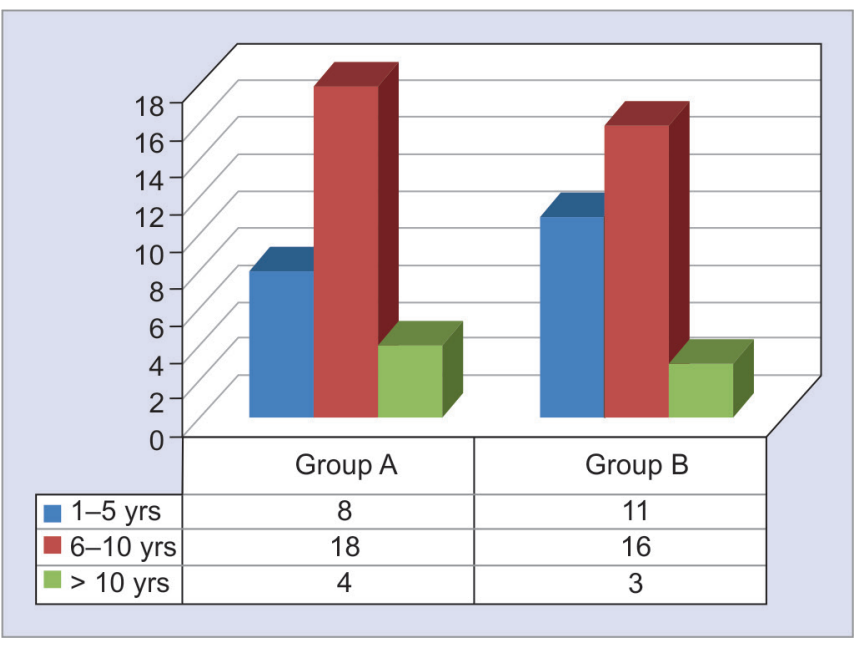

Fig. 2: Duration of infertility in the studied cases

Table 2: Mean age of the studied cases

\begin{tabular}{llll}
\hline Study group & Mean age (years) & Std. deviation & Significance \\
\hline Group A & 7.43 & 2.50 & $P=0.44$ \\
Group B & 6.93 & 2.54 & Not significant \\
\hline
\end{tabular}

and 10 years, whereas 8 patients (29.16\%) had infertility of $1-5$ years. Four patients (20.84\%) had infertility of more than 10 years. Among group B patients, 16 patients (53.33\%) had infertility of 6-10 years followed by $1-5$ years (36.66\%) and infertility of more than 10 years (10\%) (Fig. 2).

The mean duration of infertility in patients of group $A$ and group $B$ was found to be $7.43 \pm 2.50$ and $6.93 \pm 2.54$ years, respectively. There was no statistically significant difference in the mean duration of infertility in patients of group A and group B (Table 2).

The most common cause of infertility in group $A$ was found to be combined factors (26.67\%), followed by tubal factors (23.33\%), ovulatory dysfunction (20\%), and unexplained infertility (16.67\%). Four (13.33\%) patients had male factors as cause of infertility. In group $B$, the most common factor responsible for infertility was found to be tubal causes (36.67\%), followed by combined factors (20\%), ovulatory dysfunction (16.67\%), and male factors (16.67\%). In group B, male factor infertility was seen in $3(10 \%)$ patients (Table 3 ).

Antral follicle count was done in all patients on D2 of the cycle. The mean number of antral follicle count in group A was $9.52 \pm 1.82$, whereas in group $B$, it was $10.42 \pm 1.52$ (Fig. 3).

There was no statistically significant difference in the mean antral follicle count in patients of group $A$ and group $B(P=0.11)$ (Table 4).

The mean endometrial thickness in group $A$ was $9.72 \pm 1.78$, whereas in group $B$, the mean endometrial thickness was found to be $9.12 \pm 1.14$ (Fig. 4).

There was no statistically significant difference in the mean endometrial thickness in patients of group $A$ and group $B(P=0.12)$ (Table 5).
Table 3: Causes of infertility in the studied cases

\begin{tabular}{lrcrrr}
\hline & \multicolumn{2}{c}{ Group $A$} & & \multicolumn{2}{c}{ Group B } \\
\cline { 2 - 3 } \cline { 5 - 6 } Causes of infertility & $N$ & $\%$ & & $N$ & $\%$ \\
\hline Ovulatory dysfunction & 6 & 20.00 & & 5 & 16.67 \\
Tubal causes & 7 & 23.33 & & 11 & 36.67 \\
Male factors & 4 & 13.33 & & 5 & 16.67 \\
Combined factors & 8 & 26.67 & & 6 & 20.00 \\
Unexplained infertility & 5 & 16.67 & & 3 & 10.00 \\
Total & 30 & 100 & & 30 & 100 \\
\hline
\end{tabular}

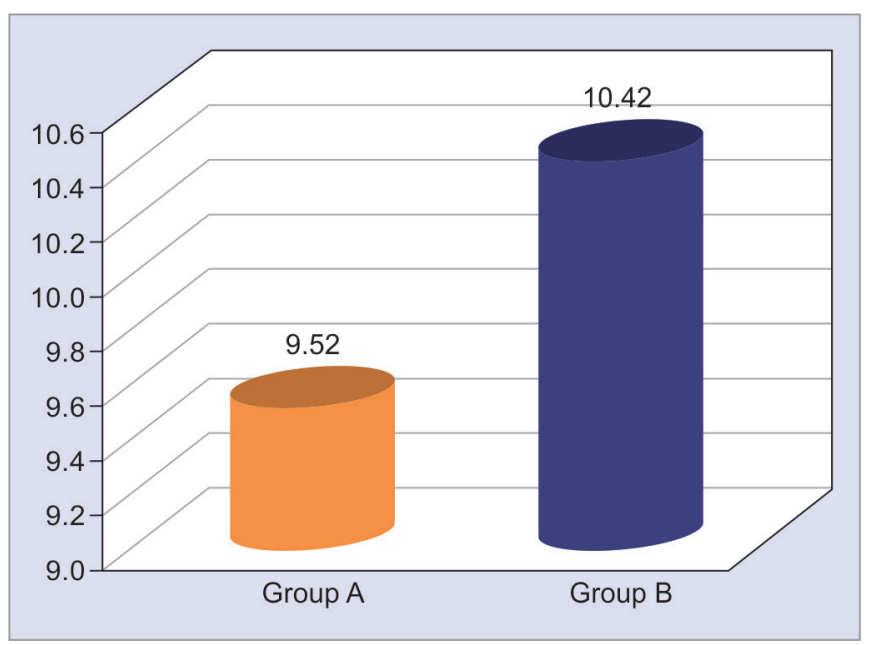

Fig. 3: Mean antral follicle rate in studied cases

Table 4: Comparison of mean follicle count in studied cases

\begin{tabular}{lcll}
\hline Study group & Mean follicle count & Std. deviation & Significance \\
\hline Group A & 9.72 & 1.82 & $P=0.11$ \\
Group B & 10.42 & 1.52 & Not significant \\
\hline
\end{tabular}

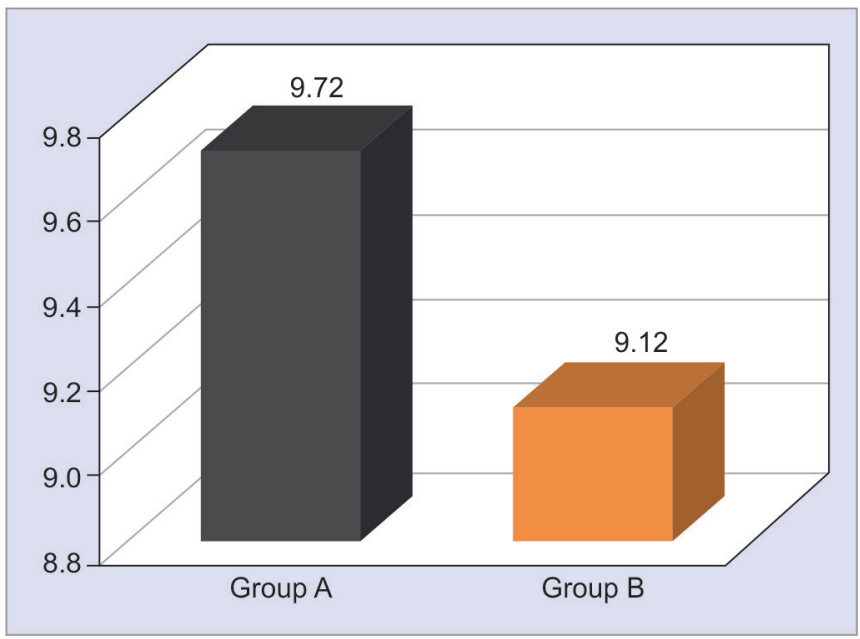

Fig. 4: Mean endometrial thickness in studied cases

The analysis of successful pregnancy rate as diagnosed on the basis of $\beta$-hCG and on fetal cardiac activity at 10 weeks was also studied in both the cases. The pregnancy rates and incidence of 
Table 5: Comparison of mean endometrial thickness in studied cases.

\begin{tabular}{llll}
\hline Study group & Mean follicle count & Std. deviation & Significance \\
\hline Group A & 9.72 & 1.78 & $P=0.12$ \\
Group B & 9.12 & 1.14 & Not significant \\
\hline
\end{tabular}

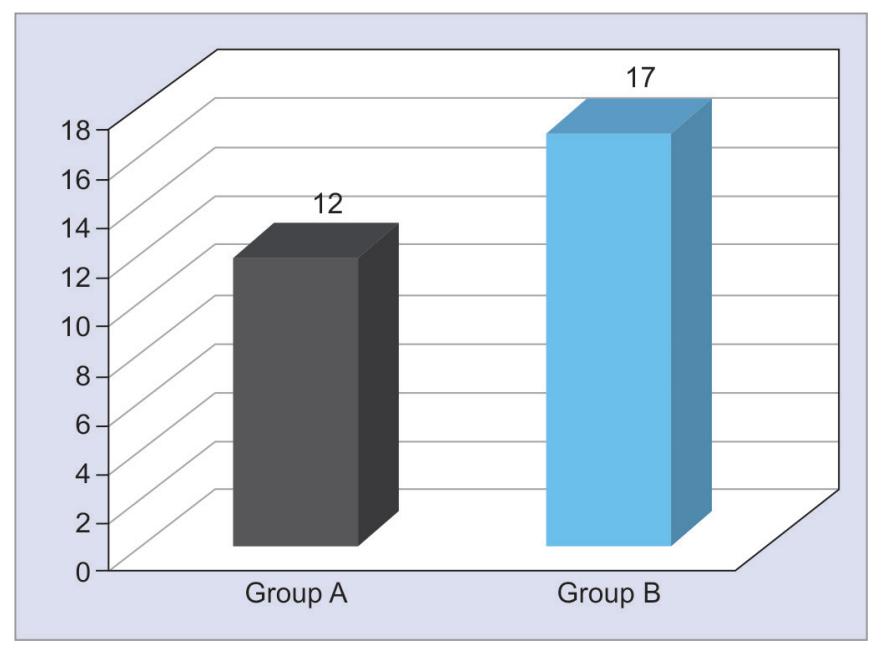

Fig. 5: Successful pregnancy rate in studied cases

Table 6: Comparison of successful pregnancy rates in studied cases

\begin{tabular}{llll}
\hline \multicolumn{4}{c}{$\begin{array}{c}\text { Successful pregnancy rate } \\
(\beta \text { - } h \text { CG on D14) }\end{array}$} \\
\cline { 2 - 3 } Study group & No. of patients & Percentage (\%) & Significance \\
\hline Group A & 12 & 40 & $P=0.301$ \\
Group B & 17 & 56.67 & Not significant \\
\hline
\end{tabular}

Table 7: Comparison of clinical pregnancy rates on the basis of fetal cardiac activity

\begin{tabular}{|c|c|c|c|}
\hline \multirow[b]{2}{*}{ Study group } & \multicolumn{2}{|c|}{$\begin{array}{c}\text { Successful pregnancy rate } \\
\text { (fetal cardiac activity) }\end{array}$} & \multirow[b]{2}{*}{ Significance } \\
\hline & No. of patients & Percentage (\%) & \\
\hline Group A & 13 & 43.33 & $P=0.30$ \\
\hline Group B & 18 & 60 & Not significant \\
\hline
\end{tabular}

abortions in both the groups were compared. Out of the 30 cases in group A, 12 (40\%) patients were confirmed to be having successful pregnancy on the basis of $\beta$-hCG levels on D14, whereas 17 (56.67\%) patients in group B were found to have successful pregnancy on the basis of $\beta$-hCG (Fig. 5).

The successful pregnancy rate as evidenced by $\beta$-hCG levels on D14 was analyzed: 12 (40\%) and 17 (56.67\%) patients were found to have successful pregnancy. Though the successful pregnancy rate was better in group $B$, the results were found to be comparable and there was no statistically significant difference in both the groups $(P=0.301)$ (Table 6).

Clinical pregnancy rates were compared in both the groups by ultrasound examination at 8 weeks. Fetal cardiac activity was present in $13(43.33 \%)$ patients and $18(60 \%)$ patients in group $A$ and group $B$, respectively. The difference was not found to be statistically significant $(P=0.30)$ (Table 7).

\section{Discussion}

We conducted this study to compare the effectiveness of uterine scratching and lateral metroplasty in patients having infertility and repeated implantation failure. The outcome measures that were compared included successful pregnancy rate and clinical pregnancy rate. Repeated implantation failure is usually defined as failure of embryos of good quality to successfully implant after several in vitro fertilization (IVF) treatment cycles. ${ }^{8}$ One of the difficulties in studying repeated implantation failure is lack of consensus on minimum number of implantation failures required to label a patient to be having repeated implantation failure although various researchers take failure of at least three cycles as criteria for diagnosing repeated implantation failure. ${ }^{9}$ Various causes of repeated implantation failure may be related to maternal or embryonal factors, and different studies have reported different etiologies responsible for repeated implantation failure. ${ }^{10}$ One of the most important mechanisms of implantation failure appears to be disturbances in endometrium at cellular and molecular level. The management of patients with repeated implantation failure depends upon the cause of such implantation failure, and one of the most important reasons of difficulties in managing such patients is lack of exact diagnosis of the cause of implantation failure. ${ }^{11}$

Deliberate therapeutic local endometrial injury is reported to improve implantation rates in subsequent cycle by many authors doing research on causes and management of repeated implantation failure. Naverkar et al. conducted a randomized controlled trial (RCT) consisting of 100 patients with previous failed IVF despite transfer of good-quality embryos. ${ }^{12}$ The purpose of the study was to determine whether endometrial injury caused by Pipelle sampling in the nontransfer cycle could improve the probability of pregnancy in the subsequent IVF cycle in patients who had previous failed IVF outcome. The authors found that live birth rate was significantly higher in the intervention group compared to control group. On the basis of these findings, the authors concluded that endometrial injury in nontransfer cycle improves the live birth rate, clinical pregnancy rates, and implantation rates in the subsequent IVF cycles. Similar advantage of endometrial scratching in patients with repeated implantation failure was reported by the authors such as Liu et al. ${ }^{13}$ and Nastri et al. ${ }^{14}$

Hysteroscopic metroplasty is another intervention, which is being increasingly used for the management of unexplained implantation failures. Haydardedeoğlu et al. conducted a retrospective cohort study of patients who had undergone metroplasty for unexplained infertility. The authors found that in the primary infertility group, the clinical pregnancy rate was $45.68 \%$ (74/162) and the live birth rate was 38.9\% (63/162), and in the secondary infertility group, the clinical pregnancy rate was $55.45 \%$ $(61 / 110)$ and the live birth rate was $49 \%$ (54/110) after metroplasty. The authors concluded that hysteroscopic metroplasty increases chances of implantation and successful pregnancy in patients with unexplained infertility. ${ }^{15}$

Despite a thorough literature review, we did not find any study comparing the endometrial scratching and hysteroscopic lateral metroplasty; hence, the results of our study could not be compared with any such comparative study. We in our study found lateral metroplasty to be having a better outcome as compared to endometrial scratching in terms of successful pregnancy rate and clinical pregnancy rate though the difference was not found to be statistically significant. 


\section{Conclusion}

Repeated implantation failure is one of the important causes of failure of in vitro fertilization treatment. The causes of repeated implantation failure can be diverse and may consist of maternal or embryonal factors. Endometrial scratching and hysteroscopic lateral metroplasty appear to be effective in increasing rates of implantations. Though lateral metroplasty appears to be having better results than endometrial scratching, the difference was not found to be statistically significant.

\section{References}

1. Laufer N, Simon A. Recurrent implantation failure: current update and clinical approach to an ongoing challenge. Fertil Steril 2012;97(5):1019-1020. DOI: 10.1016/j.fertnstert.2012.03.033.

2. Bhattacharya S, Maheshwari A, Mollison J. Factors associated with failed treatment: an analysis of 121,744 women embarking on their first IVF cycles. PLoS One 2013;8(12):e82249. DOI: 10.1371/journal.pone.0082249.

3. Urman B, Yakin K, Balaban B. Recurrent implantation failure in assisted reproduction: how to counsel and manage. A. General considerations and treatment options that may benefit the couple. Reprod Biomed Online 2005;11(3):371-381. DOI: 10.1016/s1472-6483(10)60846-2.

4. Larsen EC, Christiansen OB, Kolte AM, et al. New insights into mechanisms behind miscarriage. BMC Med 2013;11:154. DOI: 10.1186/1741-7015-11-154.

5. Findikli N, Kahraman S, Saglam Y, et al. Embryo aneuploidy screening for repeated implantation failure and unexplained recurrent miscarriage. Reprod Biomed Online 2006;13(1):38-46. DOI: 10.1016/ s1472-6483(10)62014-7.

6. Barash A, Dekel N, Fieldust S, et al. Local injury to the endometrium doubles the incidence of successful pregnancies in patients undergoing in vitro fertilization. Fertil Steril 2003;79(6):1317-1322. DOI: 10.1016/s0015-0282(03)00345-5.

7. Barranger E, Gervaise A, Doumerc S, et al. Reproductive performance after hysteroscopic metroplasty in the hypoplastic uterus: a study of 29 cases. BJOG 2002;109(12):1331-1334. DOI: 10.1046/j.14710528.2002.01448.x.

8. Coughlan C, Ledger W, Wang $Q$, et al. Recurrent implantation failure: definition and management. Reprod Biomed Online 2014;28(1):14-38. DOI: 10.1016/j.rbmo.2013.08.011.

9. Rinehart J. Recurrent implantation failure: definition. J Assist Reprod Genet 2007;24(7):284-287. DOI: 10.1007/s10815-007-9147-4.

10. Simon A, Laufer N. Repeated implantation failure: clinical approach. Fertil Steril 2012;97(5):1039-1043. DOI: 10.1016/j. fertnstert.2012.03.010.

11. Margalioth EJ, Ben-Chetrit A, Gal M, et al. Investigation and treatment of repeated implantation failure following IVF-ET. Hum Reprod 2006;21(12):3036-3043. DOI: 10.1093/humrep/del305.

12. Narvekar SA, Gupta N, Shetty N, et al. Does Local endometrial injury in the nontransfer cycle improve the IVF-ET outcome in the subsequent cycle in patients with previous unsuccessful IVF? A randomized controlled pilot study. J Hum Reprod Sci 2010;3(1):15-19. DOI: 10.4103/0974-1208.63116.

13. Liu W, Tal R, Chao H, et al. Effect of local endometrial injury in proliferative vs. luteal phase on IVF outcomes in unselected subfertile women undergoing in vitro fertilization. Reprod Biol Endocrinol 2017;15(1):75. DOI: 10.1186/s12958-017-0296-8.

14. Nastri CO, Polanski LT, Raine-Fenning N, et al. Endometrial scratching for women with repeated implantation failure. Hum Reprod 2014;29(12):2855-2856. DOI: 10.1093/humrep/deu257.

15. Haydardedeoğlu B, Doğan Durdağ G, Şimşek S, et al. Reproductive outcomes of office hysteroscopic metroplasty in women with unexplained infertility with dysmorphic uterus. Turk J Obstet Gynecol 2018;15(3):135-140. DOI: 10.4274/tjod.30111. 